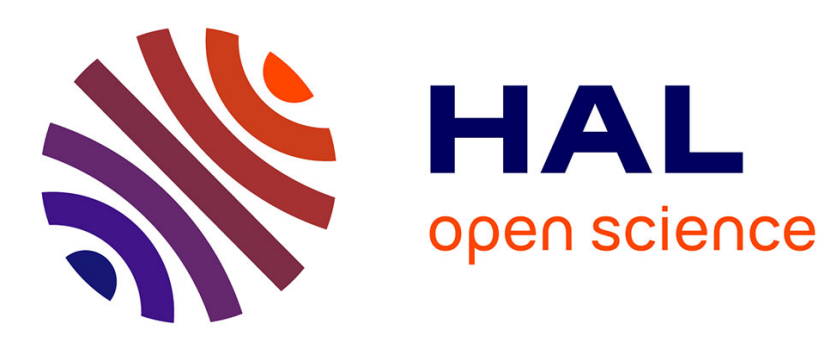

\title{
On emulated nonlinear reduced-order observers for networked control systems
}

Romain Postoyan, Dragan Nesic

\section{To cite this version:}

Romain Postoyan, Dragan Nesic. On emulated nonlinear reduced-order observers for networked control systems. Automatica, 2012, 48 (4), pp.645-652. 10.1016/j.automatica.2012.01.017 . hal-00675234

\section{HAL Id: hal-00675234 \\ https://hal.science/hal-00675234}

Submitted on 22 Dec 2012

HAL is a multi-disciplinary open access archive for the deposit and dissemination of scientific research documents, whether they are published or not. The documents may come from teaching and research institutions in France or abroad, or from public or private research centers.
L'archive ouverte pluridisciplinaire HAL, est destinée au dépôt et à la diffusion de documents scientifiques de niveau recherche, publiés ou non, émanant des établissements d'enseignement et de recherche français ou étrangers, des laboratoires publics ou privés. 


\title{
On emulated nonlinear reduced-order observers for networked control systems
}

\author{
Romain Postoyan ${ }^{\mathrm{a}}$, Dragan Nešić ${ }^{\mathrm{b}}$, \\ ${ }^{a}$ Centre de Recherche en Automatique de Nancy, UMR 7039, Nancy-Université, CNRS, France \\ ${ }^{\mathrm{b}}$ Department of Electrical and Electronic Engineering, The University of Melbourne, Parkville VIC 3010, Australia
}

\begin{abstract}
We consider a general class of nonlinear reduced-order observers and show that the global asymptotic convergence of the observation error in the absence of network-induced constraints is maintained for the emulated observer semiglobally and practically (with respect to the maximum allowable transmission interval) when system measurements are sent through a communication channel. Networks governed by a Lyapunov uniformly globally asymptotically stable protocol are investigated. Our results can be used to synthesize various observers for networked control systems for a range of network configurations, as we illustrate it by considering classes of immersion and invariance observers which include the circle-criterion observers.
\end{abstract}

Key words: Networked control systems, observers, nonlinear, stability

\section{Introduction}

Networked control systems (NCS) refer to systems for which communication between the controller and spatially distributed sensors / actuators is ensured via a shared network channel. Due to their great flexibility, low cost and easy maintenance, NCS have become prevalent in many emerging control applications such as driveby-wire cars and fly-by-wire aircrafts. However, the communication constraints induced by the network imply additional difficulties compared to classical control systems. When considering the observer design problem, one of the main issues is due to the scheduling: only a subset of sensors is allowed to send their data to the observer at the transmission instants. The sporadic and partial availability of system measurements, which are respectively characterized by the maximum allowable transmission interval (MATI) and the scheduling protocol, requires the development of appropriate observer designs.

\footnotetext{
* This work was supported by the Australian Research Council under the Future Fellowship and Discovery Grants Schemes and was done while R. Postoyan was a research assistant at the Electrical and Electronic Department of the University of Melbourne. This paper was not presented at any IFAC meeting. Corresponding author R. Postoyan. Tel. $+33(0) 3.83 .59 .56 .47$. Fax +33(0)3.83.59.56.44.
}

Email addresses: romain.postoyan@cran.uhp-nancy.fr (Romain Postoyan), d.nesic@ee.unimelb.edu.au (Dragan Nešić).
A framework for the synthesis of full order observers for nonlinear NCS has been proposed in [16], via an emulation-like approach, that encompasses the methods proposed in $[8,15]$ as particular cases. Provided that the continuous-time observer is sufficiently robust to measurement errors, sufficient conditions are given to guarantee the global convergence of the observation error for various in-network processing implementations and Lyapunov uniformly globally exponentially stable (UGES) protocols. Computable MATI bounds are obtained and our results have been applied to derive linear observers [16] and high-gain observers [14] for NCS for a range of network configurations. This work has then been extended to larger classes of systems and protocols in [17], by allowing Lyapunov UGAS (uniformly globally asymptotically stable) protocols as introduced in [13], and by assuming that all the input-to-state stability (ISS) assumptions in [16] hold with nonlinear gains (and not linear gains). As a consequence, the observation error is no longer ensured to converge globally in the presence of network, but semiglobally (and practically) with respect to the MATI. Obtained results have been applied to build circle-criterion observers ([2]) for NCS.

In this paper, we study the emulation of reduced-order observers for NCS. To the best of our knowledge, it is the first time that reduced-order observers are built for NCS subject to scheduling. We start by considering a continuous-time observer design which comes from im- 
mersion and invariance techniques $[9,3]$ and which allows one to recover many observer syntheses as mentioned in [9], such as linear Luenberger observers, highgain observers $[10,5]$, observers for linear systems up to an output injection [11,7] and circle-criterion observers [2] to mention a few. A model is then derived for the observer design for NCS, which is based on a different set of coordinates compared to $[16,17]$. We show that if the continuous-time observer is built to ensure some ISS properties for the observation error while ignoring the network, then this property will be maintained semiglobally and practically w.r.t. the parameter MATI, when the system measurements are sent through a network controlled by a Lyapunov UGAS protocol, under mild conditions. We use the obtained results to build up a class of immersion and invariance observers as well as circle-criterion observers for NCS.

\section{Preliminaries}

A function $\gamma: \mathbb{R}_{>0} \rightarrow \mathbb{R}_{>0}$ is of class $\mathcal{K}$ if it is continuous, zero at zero and strictly increasing and is of class $\mathcal{K}_{\infty}$ if, in addition, it is unbounded. By extension, for $\gamma: \mathbb{R}_{\geq 0}^{2} \rightarrow \mathbb{R}_{\geq 0}$ is of class $\mathcal{K} \mathcal{K}$ if, for any $\left(s_{1}, s_{2}\right) \in \mathbb{R}_{\geq 0}^{2}$, $\gamma\left(s_{1}, \cdot\right)$ and $\gamma\left(\cdot, s_{2}\right)$ are of class $\mathcal{K}$. A continuous function $\gamma: \mathbb{R}_{\geq 0}^{2} \longrightarrow \mathbb{R}_{\geq 0}$ is of class $\mathcal{K} \mathcal{L}$ if for each $t \in \mathbb{R}_{\geq 0}$, $\gamma(\cdot, t)$ is of class $\mathcal{K}$, and, for each $s \geq 0, \gamma(s, \cdot)$ is decreasing to zero. Let $C\left(\mathbb{R}^{p}, \mathbb{R}^{q}\right)$ and $C^{1}\left(\mathbb{R}^{p}, \mathbb{R}^{q}\right)$ respectively denote the space of all continuous and continuously differentiable mappings from $\mathbb{R}^{p}$ to $\mathbb{R}^{q}$. The Euclidean norm of a vector or a matrix is denoted by $|\cdot|$. Given a measurable, locally essentially bounded signal $f:\left[t_{0}, \infty\right) \rightarrow \rightarrow \mathbb{R}^{n}$, we denote its $\mathcal{L}_{\infty}$ norm as $\|f\|_{\infty}=$ ess. $\sup _{\tau \geq t_{0}}|f(\tau)|$. When $\|f\|_{\infty}$ is bounded, we write that $f \in \mathcal{L}_{\infty}$ and we say that $f \in \mathcal{L}_{\text {loc, } \infty}$, when for any $t_{0} \leq t_{1} \leq t_{2}<\infty$, ess. $\sup _{t_{2} \geq \tau \geq t_{1}}|f(\tau)|$ is bounded. For $(x, y) \in \mathbb{R}^{n+m}$, the notation $(x, y)$ stands for $\left[x^{\mathrm{T}}, y^{\mathrm{T}}\right]^{\mathrm{T}}$ and II denotes the identity matrix of appropriate dimensions. A mapping $h(t, z, y): \mathbb{R}_{\geq 0} \times \mathbb{R}^{n_{z}+n_{y}} \rightarrow \mathbb{R}^{n_{\eta}}$ is said to be left-invertible w.r.t. its second argument if there exists a mapping $h^{L}: \mathbb{R}_{>0} \times \mathbb{R}^{n_{\eta}+n_{y}} \rightarrow \mathbb{R}^{n_{z}}$ such that $h^{L}(t, h(t, z, y), y)=z$ for all $t, z, y$. Consider the system:

$$
\dot{x}=f(t, x, u), \quad y=h(t, x),
$$

where $x \in \mathbb{R}^{n_{x}}$ is the state, $y \in \mathbb{R}^{n_{y}}$ the output and $u \in \mathbb{R}^{n_{u}}$ the input.

Definition 1 System (1) is said to be uniformly bounded-input-bounded-state (UBIBS) with input $u$ if there exist $K, \gamma \in \mathcal{K}$, such that, for any $x_{0} \in \mathbb{R}^{n_{x}}$, $u \in \mathcal{L}_{\infty}:|x(t)| \leq K\left(\left|x_{0}\right|\right)+\gamma\left(\|u\|_{\infty}\right)$ for all $t \geq t_{0} \geq 0$. When $u \equiv 0$, we say that it is uniformly globally stable (UGS).

Definition 2 System (1) is said to be uniformly forward complete with input $u$ if there exist $\nu_{1}, \nu_{2}, \nu_{3} \in \mathcal{K}$ and $c \in$
$\mathbb{R}_{\geq 0}$ such that, for any $x_{0} \in \mathbb{R}^{n_{x}}, u \in \mathcal{L}_{\infty}$, along solutions to $(1):|x(t)| \leq \nu_{1}\left(t-t_{0}\right)+\nu_{2}\left(\left|x_{0}\right|\right)+\nu_{3}\left(\|u\|_{\infty}\right)+c$ for all $t \geq t_{0} \geq 0$.

Remark 3 Definition 2 is inspired by [1] (in particular Corollary 2.3) where forward completeness characterizations are proposed for time-invariant systems.

\section{System models}

We pursue the emulation-like approach for the observer design for NCS like in $[16,17]$, which is originally inspired by the work of $[18,12]$ for the control of NCS. The approach consists in synthesizing an observer while ignoring the communication constraints, afterwards the networked-induced errors are taken into account.

\subsection{Immersion and invariance reduced-order observers}

We choose to focus on observer designs which come from immersion and invariance techniques [9,3] because a number of observer constructions available in the literature can then be considered in a unified manner. Consider the plant modeled by the following continuous-time equations:

$$
\dot{\eta}=f_{\eta}(t, \eta, y, w), \quad \dot{y}=f_{y}(t, \eta, y, w),
$$

where $\eta \in \mathbb{R}^{n_{\eta}}$ is the unmeasured part of the state, $y \in \mathbb{R}^{n_{y}}$ is the measurable part of the state, $w \in \mathbb{R}^{n_{w}}$ is an exogenous disturbance input. For the purpose of constructing an observer which estimates $\eta$, we may employ a coordinate transformation, thus we define the variable $z=T_{P}(t, \eta, y) \in \mathbb{R}^{n_{z}}$ where $n_{z} \geq n_{\eta}$ and $T_{P} \in C^{1}\left(\mathbb{R}_{\geq 0} \times \mathbb{R}^{n_{\eta}+n_{y}}, \mathbb{R}^{n_{z}}\right)$ is a left-invertible mapping in its second argument with left-inverse $T_{P}^{L}$. We denote:

$$
\dot{z}=f_{z}(t, z, y, w), \quad \dot{y}=f_{y}\left(t, T_{P}^{L}(t, z, y), y, w\right),
$$

where

$$
\begin{aligned}
f_{z}(t, z, y, w) & =\frac{\partial T_{P}}{\partial t}\left(t, T_{P}^{L}(t, z, y), y\right) \\
+ & \frac{\partial T_{P}}{\partial \eta}\left(t, T_{P}^{L}(t, z, y), y\right) f_{\eta}\left(t, T_{P}^{L}(t, z, y), y, w\right) \\
+ & \frac{\partial T_{P}}{\partial y}\left(t, T_{P}^{L}(t, z, y), y\right) f_{y}\left(t, T_{P}^{L}(t, z, y), y, w\right) .
\end{aligned}
$$

The idea is to design a reduced-order observer for system (3) that will provide us with an estimate of $z$, namely $\bar{z}$. The estimate of $\eta$, denoted $\bar{\eta}$, will then be obtained by using $\bar{\eta}=T_{P}^{L}(t, \bar{z}, y)$. In a number of cases, $n_{z}$ is equal to $n_{\eta}$. Nonetheless, it is known that immersing the plant (2) into a system of larger dimension $\left(n_{z}>n_{\eta}\right)$ may help for designing an observer (see Chapter 5 in [3] and the references therein). Now to show the observation error $\eta-\bar{\eta}$ satisfies some ISS properties, we need to assume that $T_{P}$ guarantees the following condition. 
Assumption 4 There exist $\theta_{P}, \bar{\theta}_{P} \in \mathcal{K}_{\infty}$ such that for any $a, \bar{a} \in \mathbb{R}^{n_{z}}, b, \bar{b} \in \mathbb{R}^{n_{y}}$ and $t \in \mathbb{R}_{\geq 0}$, this holds: $\left|T_{P}^{L}(t, a, b)-T_{P}^{L}(t, \bar{a}, \bar{b})\right| \leq \theta_{P}(|a-\bar{a}|)+\overline{\bar{\theta}}_{P}(|b-\bar{b}|)$.

The reduced-order observer takes the following form:

$$
\left.\begin{array}{l}
\dot{\bar{q}}=f_{\bar{q}}(t, \bar{q}, y) \\
\bar{z}=T_{O}(t, \bar{q}, y) \\
\bar{\eta}=T_{P}^{L}(t, \bar{z}, y)
\end{array}\right\}
$$

where $\bar{q} \in \mathbb{R}^{n_{\bar{q}}}$ is the observer state $\left(n_{\bar{q}}=n_{z}\right), \bar{z} \in \mathbb{R}^{n_{z}}$ is defined through the output map $T_{O} \in C^{1}\left(\mathbb{R}_{\geq 0} \times\right.$ $\left.\mathbb{R}^{n_{\bar{q}}+n_{y}}, \mathbb{R}^{n_{z}}\right)$, where $T_{O}$ is left-invertible w.r.t. its second argument with left-inverse $T_{O}^{L}$, and $T_{P}^{L}$ is used to obtain $\bar{\eta} \in \mathbb{R}^{n_{\eta}}$, the estimate of $\eta$. Variable $\bar{z}$ may seem superfluous since $\bar{\eta}=T_{P}^{L}\left(t, T_{O}(t, \bar{q}, y), y\right)$ according to (4). Nevertheless, it is sometimes necessary to analyse the dynamics of $z-\bar{z}$ rather than directly those of $\eta-\bar{\eta}$ in order to deduce stability properties for the observation error, as discussed in Remark 1 in [9].

Beside the immersion and invariance observers developed in [9] and in Chapter 5 in [3], the observer formulation (4) allows one to consider various reduced-order observer designs available in the literature, such as the circle criterion observers developed in [2] as shown below.

Example 5 [Circle-criterion observers in [2]] Consider the system:

$$
\begin{aligned}
& \dot{y}=A_{1} \eta+G_{1} \gamma\left(H_{1} y+H_{2} \eta\right)+\rho_{1}(y) \\
& \dot{\eta}=A_{2} \eta+G_{2} \gamma\left(H_{1} y+H_{2} \eta\right)+\rho_{2}(y)
\end{aligned}
$$

where $A_{1}, A_{2}$ are real matrices of appropriate dimension, $\rho_{1}, \rho_{2}, \gamma$ are continuous functions and $\gamma$ satisfies some monotonic properties. The following reduced-order observer is built:

$$
\begin{aligned}
\dot{\bar{q}}= & \left(A_{2}+N A_{1}\right) \bar{q}+\left(G_{2}+N G_{1}\right) \\
& \times \gamma\left(H_{2} \bar{q}+\left(H_{1}-H_{2} N\right) y\right)+\bar{\rho}(y) \\
\bar{\eta}= & \bar{q}-N y
\end{aligned}
$$

where $\bar{q} \in \mathbb{R}^{n_{\eta}}, \bar{\rho}(y)=N \rho_{1}(y)+\rho_{2}(y)-\left(A_{2}+N A_{1}\right) N y$ and the real matrix $N$ satisfies a given $L M I$ (see (30) in Section 5.2). We have: $z=\eta+N y, T_{P}(t, \eta, y)=\eta+N y$ (that satisfies Assumption 4), $\bar{z}=\bar{q}$ i.e. $T_{O}(t, \bar{q}, y)=\bar{q}$.

\subsection{NCS model}

When the system output is transmitted through a serial communication channel, $y$ is no longer available to the observer but $\hat{y}$, which is generated from the most recent system output sent through the network, (see $[12,16]$ for instance, where a similar approach is followed and discussed in more details). The problem can then be modeled as follows:

$$
\left.\begin{array}{rlrl}
\dot{z} & =f_{z}(t, z, y, w) & & \forall t \in\left[t_{i-1}, t_{i}\right] \\
\dot{y} & =f_{y}\left(t, T_{P}^{L}(t, z, y), y, w\right) & & \forall t \in\left[t_{i-1}, t_{i}\right] \\
\dot{\bar{q}} & =f_{\bar{q}}(t, \bar{q}, \hat{y}) & & \forall t \in\left[t_{i-1}, t_{i}\right] \\
\bar{z} & =T_{O}(t, \bar{q}, \hat{y}) & & \\
\bar{\eta} & =T_{P}^{L}(t, \bar{z}, \hat{y}) & & \\
\dot{\hat{y}} & =\hat{f}_{y}(t, \hat{y}, \bar{z}) & & \\
\hat{y}\left(t_{i}^{+}\right) & =y\left(t_{i}\right)+h\left(i, e\left(t_{i}\right)\right) & &
\end{array}\right\}
$$

where $e=\hat{y}-y \in \mathbb{R}^{n_{e}}\left(n_{e}=n_{y}\right)$ denotes the networkinduced error and the sequence $t_{i}, i \in \mathbb{Z}_{>0}$, of monotonically increasing transmission times satisfies $v \leq t_{i}-$ $t_{i-1} \leq \tau$ for some fixed ${ }^{1} v \in \mathbb{R}_{>0}, \tau \in[v, \infty)$ is the MATI, $t_{0} \in \mathbb{R}_{\geq 0}$ being the initial time. The setup is the following (see Figure 1). Sensors are grouped into $l$ sensor nodes, which are connected to the communication channel. At each transmission instant, a unique node is designated by the protocol to transmit its data: typically, by decomposing the system output into $l$ corresponding subvectors $y=\left(y_{1}, \ldots, y_{l}\right)$, if node $j$ has been selected at time $t_{i}$ the corresponding subvector of $\hat{y}$ is set to $\hat{y}_{j}\left(t_{i}^{+}\right)=y_{j}\left(t_{i}\right)$ while the others remain unchanged. This mechanism is modeled by function $h$. The network may also contain an arbitrary number of passive nodes which can only receive packets. Without loss of generality, we suppose that there is only one passive node where the observer is located. Function $\hat{f}_{y} \bmod -$ els the in-network processing algorithm, i.e. the way $\hat{y}$ is generated between the transmission instants. In many problems, zero-order-hold ( $\mathrm{ZOH})$ algorithms are considered that is: $\hat{f}_{y}=0$, namely the variable $\hat{y}$ is kept constant between two transmission instants. Nevertheless, our approach allows us to study a range of innetwork processing algorithms, like in $[12,16,17]$, such as the following predictive-type implementation introduced in [8] for the design of sampled-data observers: $\hat{f}_{y}(t, \hat{y}, \bar{z})=f_{y}(t, \bar{\eta}, \hat{y}, 0)=f_{y}\left(t, T_{P}^{L}(t, \bar{z}, \hat{y}), \hat{y}, 0\right)$.

We write system $(9)$ in the coordinates $(\zeta, \chi, e)$ where $\zeta=z-\bar{z} \in \mathbb{R}^{n_{\zeta}}, \chi=(z, y) \in \mathbb{R}^{n_{\chi}}$ and we introduce the observation error $\xi=\eta-\bar{\eta} \in \mathbb{R}^{n_{\xi}}$ :

$$
\begin{aligned}
\dot{\zeta} & =f_{\zeta}(t, \zeta, \chi, e, w) & & \forall t \in\left[t_{i-1}, t_{i}\right] \\
\dot{\chi} & =f_{\chi}(t, \chi, w) & & \forall t \in\left[t_{i-1}, t_{i}\right] \\
\dot{e} & =g(t, \zeta, \chi, e, w) & & \forall t \in\left[t_{i-1}, t_{i}\right] \\
\zeta\left(t_{i}^{+}\right) & =\zeta\left(t_{i}\right) & & \\
\chi\left(t_{i}^{+}\right) & =\chi\left(t_{i}\right) & & \\
e\left(t_{i}^{+}\right) & =h\left(i, e\left(t_{i}\right)\right) & & \\
\xi & =h_{\xi}(t, \zeta, \chi, e), & &
\end{aligned}
$$

1 Note that $v$ is arbitrary and it is used to prevent Zeno solutions in (9), see [12]. 


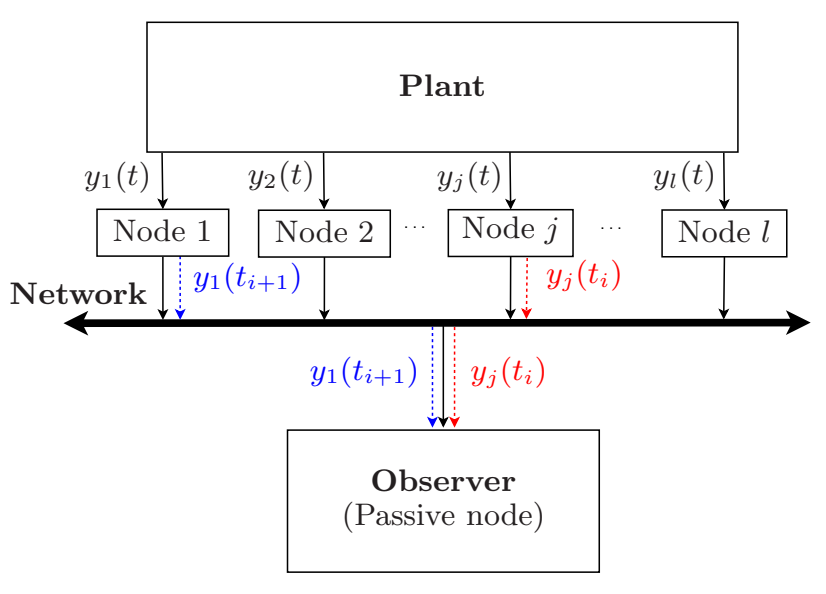

Fig. 1. Block diagram of the NCS

where $f_{\zeta}(t, \zeta, \chi, e, w)=f_{z}(t, z, y, w)-\frac{\partial T_{O}}{\partial t}\left(t, T_{O}^{L}(t, z-\right.$ $\zeta, y+e), y+e)-\frac{\partial T_{O}}{\partial \bar{q}}\left(t, T_{O}^{L}(t, z-\zeta, y+e), y+\right.$ e) $f_{\bar{q}}\left(t, T_{O}^{L}(t, z-\zeta, y+e), y+e\right)-\frac{\partial T_{Q}}{\partial \hat{y}}\left(t, T_{O}^{L}(t, z-\right.$ $\zeta, y+e), y+e) \hat{f}_{y}(t, y+e, z-\zeta), f_{\chi}(t, \chi, w)=$ $\left(f_{z}(t, z, y, w), f_{y}\left(t, T_{P}^{L}(t, z, y), y, w\right)\right), g(t, \zeta, \chi, e, w)=$ $\hat{f}_{y}(t, y+e, z-\zeta)-f_{y}\left(t, T_{P}^{L}(t, z, y), y, w\right), h_{\xi}(t, \zeta, \chi, e)=$ $T_{P}^{L}(t, z, y)-T_{P}^{L}(t, z-\zeta, y+e)$.

Remark 6 Compared to [16,17] where system (9) is written using the variables $(\xi, \bar{z}, e)$, our choice of coordinates is motivated by the fact that, when dealing with reduced-order observers, convergence properties are usually not directly shown to hold for $\xi$ but for $\zeta$. It has to be noticed that this set of coordinates simplifies the structure of the interconnections of system (10)-(16) compared to system (10)-(15) in [16] and system (14)-(19) in [17], since subsystem (11), (14) is in cascade with the others.

By ensuring appropriate stability properties for system (10), we will be able to derive stability properties for the observation error $\xi$ using Assumption 4. Equation (11) models the dynamics of the variable $(z, y)$ and is closely related to the dynamics of the plant (2) as we show it in Section 4. We refer to (15) as the protocol. It was shown in [12] that static protocols such as the roundrobin protocol (RR) (that grants access to each node at a period $l$ ), and dynamic protocols such as the try-oncediscard protocol (TOD) [18] (that gives access to the node where the error $\left|e_{j}\right|=\left|y_{j}-\hat{y}_{j}\right|$ is the biggest) can be modeled in this manner. It is also interesting to note that (15) can be used to model sampled-data systems by setting the number of nodes $l$ to 1 , this gives: $h=0$. The stability of protocol (15) has been investigated for the first time in [12], where the notion of Lyapunov UGES protocol has been introduced and then extended in [13] as follows.

Definition 7 Protocol (15) is said to be Lyapunov UGAS if there exist a function $W: \mathbb{R}_{\geq 0} \times \mathbb{R}^{n_{e}} \rightarrow \mathbb{R}_{\geq 0}$, $\rho \in[0,1)$ and $\underline{\alpha}, \bar{\alpha} \in \mathcal{K}_{\infty}$ such that, for all $i \in \mathbb{Z}_{\geq 0}$ and $e \in \mathbb{R}^{n_{e}}:$

$$
\begin{aligned}
\underline{\alpha}(|e|) & \leq W(i, e) \leq \bar{\alpha}(|e|), \\
W(i+1, h(i, e)) & \leq \rho W(i, e) .
\end{aligned}
$$

We say that protocol (15) is Lyapunov UGES, if, in addition, functions $\underline{\alpha}, \bar{\alpha}$ are linear.

It has been shown in Propositions 4 and 5 in [12] that RR and TOD protocols and, as a consequence the sampleddata case, are Lyapunov UGES. In [13], modified versions of the RR and TOD protocols have been developed which have the interesting features to transmit, respectively, less frequently and less information as the network-induced error $e$ approaches the origin. Both have been shown to be Lyapunov UGAS (see Examples $1-2$ in [13]).

Remark 8 When the observer is implemented using a dynamic protocol i.e. when the schedule of the sensor nodes transmissions is not fixed (like the (modified) TOD protocol and the modified $R R$ protocol) with in-network processing algorithms that require the knowledge of $\bar{z}$ or $\bar{\eta}$ (like the predictive-type in-network processing algorithm), the use of smart sensors (i.e. sensors which have sufficient computational capacities to run the algorithm) is required as explained in Section III.C.2 in [16].

The main focus of this study is to guarantee the convergence of the observation error $\xi$ when the system output is transmitted through a serial communication channel ruled by a Lyapunov UGAS protocol.

\section{Main results}

In this section, we suppose that the observer (4) has been designed to guarantee an ISS property in the absence of network. We then derive sufficient conditions on the network configuration (in terms of the protocol, the MATI and the in-network processing algorithm), the observer and the plant which ensure that this stability property is maintained (semiglobally and practically) in the presence of a communication channel. The stability analysis is similar to [13] and the proofs are given in Appendix A.1.

First, we show that the $e$-system satisfies some semiglobal practical stability properties under the conditions that the protocol is Lyapunov UGAS, that the vector $g$ (which is used to define the dynamics of $e$ between the transmissions instants) is bounded (on compacts) and that transmissions occur sufficiently frequently (i.e. the MATI is small). In that way, loosely speaking, the protocol (15) is able to compensate for the destabilizing effect of the continuous-time dynamics (12) of the $e$-system. That is what is presented in the next theorem which corresponds to Theorem 1 in [13]. 
Theorem 9 Consider system (12), (15) and suppose the following.

(i) Protocol (15) is Lyapunov UGAS with a Lyapunov function $W(i, e)$ which is continuous in e, uniformly in $i$.

(ii) $g(t, \zeta, \chi, e, w)$ is bounded on compact sets, uniformly in $t$.

Then there exist $\tau^{*}, \beta_{1} \in \mathcal{K} \mathcal{L}$ and $\gamma_{1} \in \mathcal{K}_{\infty}$ such that, for any strictly positive pair $(\Delta, \varepsilon), \tau \in\left[v, \tau^{*}(\varepsilon, \Delta)\right)$ and $\max \left\{\|\zeta\|_{\infty},\|\chi\|_{\infty},\|w\|_{\infty},\left|e_{0}\right|\right\} \leq \Delta$, solutions to (12), (15) satisfy:

$$
|e(t)| \leq \beta_{1}\left(\left|e_{0}\right|, \frac{t-t_{0}}{\tau}\right)+\gamma_{1}(\varepsilon) \quad \forall t \geq t_{0} \geq 0
$$

From [12,13], the (modified) RR and the (modified) TOD protocols satisfy condition (i) of Theorem 9 . When facing difficulties for building an appropriate Lyapunov function $W$, Proposition 2 in [13] or equation (8) in [13] may be utilized to check whether condition (i) of Theorem 9 holds or not. Condition (ii) is related to the $y$-dynamics of the plant and the choice of the in-network processing implementation in view of the definition of $g$ (see Section 3.2). Before stating the main theorem of this section, we introduce the following auxiliary system which models system (10) when $e \equiv 0$ :

$$
\dot{\tilde{\zeta}}=f_{\zeta}(t, \tilde{\zeta}, \chi, 0, w)
$$

We are now ready to state the main results.

Theorem 10 Consider system (10)-(16) and suppose that the following conditions hold.

(i) Assumption 4 is satisfied.

(ii) All conditions of Theorem 9 hold.

(iii) There exist $L \in \mathcal{K}$ and $M \in \mathcal{K} \mathcal{K}$ such that for each $c>0, \max \{|\zeta|,|\bar{\zeta}|,|\chi|,|e|,|w|\}<c$ implies $\left|f_{\zeta}(t, \chi, \zeta, e, w)-f_{\zeta}(t, \chi, \bar{\zeta}, 0, w)\right| \leq L(c+1)|\zeta-\bar{\zeta}|+$ $M(c+1,|e|)$.

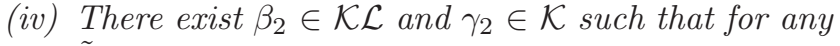
$\tilde{\zeta}_{0} \in \mathbb{R}^{n_{\zeta}}$ and $(\chi, w) \in \mathcal{L}_{\infty}$, the solutions of (20) satisfy, for all $t \geq t_{0} \geq 0$ :

$$
|\tilde{\zeta}(t)| \leq \beta_{2}\left(\left|\tilde{\zeta}_{0}\right|, t-t_{0}\right)+\gamma_{2}\left(\|w\|_{\infty}\right)
$$

(v) System (11) is UBIBS with input $w$.

Then there exist $\tau^{*}, \beta \in \mathcal{K} \mathcal{L}, \gamma \in \mathcal{K}$ such that for any strictly positive pair $(\Delta, \varepsilon), \tau \in\left[v, \tau^{*}(\varepsilon, \Delta)\right)$, $\left(\zeta_{0}, \chi_{0}, e_{0}\right) \in \mathbb{R}^{n_{\zeta}+n_{\chi}+n_{e}}$ and $w \in \mathcal{L}_{\infty}$ with $\max \left\{\left|\zeta_{0}\right|\right.$, $\left.\left|\chi_{0}\right|,\left|e_{0}\right|,\|w\|_{\infty}\right\}<\Delta$ solutions to (10)-(16) satisfy, for any $t \geq t_{0} \geq 0$ :

$$
\begin{aligned}
|e(t)| & \leq \beta_{1}\left(\left|e_{0}\right|, \frac{t-t_{0}}{\tau}\right)+\varepsilon \\
|\zeta(t)| & \leq \beta_{2}\left(\left|\zeta_{0}\right|, t-t_{0}\right)+\gamma_{2}\left(\|w\|_{\infty}\right)+\varepsilon \\
|\xi(t)| & \leq \beta\left(\left|\left(\zeta_{0}, e_{0}\right)\right|, t-t_{0}\right)+\gamma\left(\|w\|_{\infty}\right)+\varepsilon
\end{aligned}
$$

Theorem 10 tells us that the ISS property ensured by the observer when there is no network (item (iv) of Theorem 10) is preserved semiglobally and practically (see (23)) with the same class- $\mathcal{K} \mathcal{L}$ function, $\beta_{2}$, and ISS gain, $\gamma_{2}$, provided that the protocol is UGAS, the MATI is small, the vector field $f_{\zeta}$ satisfies some Lipschitz properties (item (iii) of Theorem 10) and the plant is bounded (item (v) of Theorem 10). Condition (i) of Theorem 10 is then used to derive the stability property (24) for the observation error $\xi$.

We note that, from items (i) and (iv) of Theorem 10 , there exist $\tilde{\beta} \in \mathcal{K} \mathcal{L}$ and $\tilde{\gamma} \in \mathcal{K}$ such that for any $\tilde{\zeta}_{0} \in$ $\mathbb{R}^{n_{\zeta}}$ and $(\chi, w) \in \mathcal{L}_{\infty}$, along solutions to (20), denoting $\tilde{\xi}=T_{P}^{L}(t, z, y)-T_{P}^{L}(t, z-\tilde{\zeta}, y)$, the following holds:

$$
|\tilde{\xi}(t)| \leq \tilde{\beta}\left(\left|\tilde{\zeta}_{0}\right|, t-t_{0}\right)+\tilde{\gamma}\left(\|w\|_{\infty}\right) \quad \forall t \geq t_{0} \geq 0
$$

In that way, Theorem 10 shows us that the observation error still converges under network-induced constraints, but semiglobally and practically in view of (24).

Various design methods can be employed to ensure condition (iv) of Theorem 10, see Chapter 5 in [3] or Section 5.2 for instance. Condition (iii) of Theorem 10 holds whenever function $f_{\zeta}$ is locally Lipschitz in $\chi, \zeta, e, w$, uniformly in $t$. Note that condition $(\mathrm{v})$ of Theorem 10 implies that the plant (2) is UBIBS in view of Definition 1 and Assumption 4. Conversely, it is possible to relate the stability of the plant to the stability of system (11) using the following lemma. Its proof follows directly from the use of Definitions 1 and 2 and Lemma 18 given in Appendix A.2.

Lemma 11 Suppose there exist $\vartheta_{P}, \bar{\vartheta}_{P}, \tilde{\theta}_{P}, \overline{\tilde{\theta}}_{P} \in \mathcal{K}_{\infty}$ such that, for any $(t, \eta, z, y) \in \mathbb{R}_{\geq 0} \times \mathbb{R}^{n_{\eta}+n_{z}+n_{y}}$, the following holds:

$$
\begin{aligned}
\left|T_{P}(t, \eta, y)\right| & \leq \vartheta_{P}(|\eta|)+\bar{\vartheta}_{P}(|y|) \\
\left|T_{P}^{L}(t, z, y)\right| & \leq \tilde{\theta}_{P}(|z|)+\overline{\tilde{\theta}}_{P}(|y|) .
\end{aligned}
$$

Then system (11) is UBIBS (uniformly forward complete) with input $w$ if and only if plant (2) is UBIBS (uniformly forward complete) with input $w$.

Remark 12 Condition (27) is obviously implied by Assumption 4 when $T_{P}^{L}(t, 0,0)=0$ for $t \in \mathbb{R}_{\geq 0}$, with $\tilde{\theta}_{P}=\theta_{P}$ and $\overline{\tilde{\theta}}_{P}=\bar{\theta}_{P}$. When $T_{P}(t, \eta, y)=\eta+\Lambda y$, where $\Lambda$ is a real matrix of appropriate dimension, we see that (26) and (27) hold with $\tilde{\theta}_{P}(s)=\vartheta_{P}(s)=s$ and $\overline{\tilde{\theta}}_{P}(s)=\bar{\vartheta}_{P}(s)=|\Lambda| s$ for $s \geq 0$.

Item (v) of Theorem 10 is typically needed when using zero-order-hold devices. When implementing other in-network processing algorithms such as the predictivetype introduced in Section 3.2, the following result may be derived which requires the $\chi$-dynamics to be uniformly forward complete only (and not bounded) like in Section 5.2 for instance. 
Theorem 13 Suppose the following holds.

(i) Assumption 4 holds.

(ii) Condition (i) of Theorem 9 holds.

(iii) There exists $\bar{g}: \mathbb{R}_{\geq 0} \times \mathbb{R}^{n_{\zeta}+n_{e}+n_{w}}$ that is bounded on compact sets, uniformly in $t$ and such that: $|g(t, \zeta, \chi, e, w)| \leq \bar{g}(t, \zeta, e, w)$ for any $(t, \zeta, \chi, e, w) \in \mathbb{R}_{>0} \times \mathbb{R}^{n_{\zeta}+n_{\chi}+n_{e}+n_{w}}$.

(iv) There exist $L \in \mathcal{K}$ and $M \in \mathcal{K} \mathcal{K}$ such that for each $c>0, \max \{|\zeta|,|\bar{\zeta}|,|e|,|w|\}<c$ and $\chi \in \mathbb{R}^{n_{\chi}}$ implies $\left|f_{\zeta}(t, \chi, \zeta, e, w)-f_{\zeta}(t, \chi, \bar{\zeta}, 0, w)\right| \leq L(c+$ 1) $|\zeta-\bar{\zeta}|+M(c+1,|e|)$.

(v) There exist $\beta_{2} \in \mathcal{K} \mathcal{L}$ and $\gamma_{2} \in \mathcal{K}$ such that for any $\tilde{\zeta}_{0} \in \mathbb{R}^{n_{\zeta}}, \chi \in \mathcal{L}_{\text {loc, } \infty}$ and $w \in \mathcal{L}_{\infty}$, the solutions of (20) satisfy, for any $t \geq t_{0} \geq 0$ :

$$
|\tilde{\zeta}(t)| \leq \beta_{2}\left(\left|\tilde{\zeta}_{0}\right|, t-t_{0}\right)+\gamma_{2}\left(\|w\|_{\infty}\right) .
$$

(vi) System (11) is uniformly forward complete with input $w$.

Then there exist $\tau^{*}, \beta \in \mathcal{K} \mathcal{L}, \gamma \in \mathcal{K}$ such that for any strictly positive pair $(\Delta, \varepsilon), \tau \in\left[v, \tau^{*}(\varepsilon, \Delta)\right)$, $\left(\zeta_{0}, \chi_{0}, e_{0}\right) \in \mathbb{R}^{n_{\zeta}+n_{\chi}+n_{e}}$ and $w \in \mathcal{L}_{\infty}$ with $\max \left\{\left|\zeta_{0}\right|,\left|e_{0}\right|\right.$, $\left.\|w\|_{\infty}\right\}<\Delta$ solutions to (10)-(16) satisfy (22)-(24).

Contrary to Theorem 10, the stability properties ensured by Theorem 13 hold with no boundedness restriction on the norm of $\chi_{0}$. The price to pay to be able to relax the condition (v) of Theorem 10 is that we can upperbound $|g|$ by a function that is independent of $\chi$ that is also bounded on compacts (see item (iii) of Theorem 13) and that item (iv) of Theorem 13 is verified. This allows us to consider the $\zeta$ - and $e$-systems independently of the $\chi$-system.

\section{Applications}

In this section, we focus on two nonlinear observer designs and apply respectively Theorems 10 and 13 to analyse the convergence of the observation error under network-induced constraints.

\subsection{A class of immersion $\&$ invariance observers}

Consider system (2) with no time-dependency and no perturbation $w$ only for the sake of simplicity. We assume that $f_{\eta}$ and $f_{y}$ are locally Lipschitz. We follow the observer design described in Section 3.1 to build a timeinvariant observer of the form of (4) where $f_{\bar{q}}$ is locally Lipschitz and the mappings $T_{P}$ and $T_{O}$ verify the following.

Assumption 14 The mappings $T_{P}^{L}, T_{O}^{L}$ and the first derivatives of $T_{P}$ and $T_{O}$ are locally Lipschitz and $T_{P}$ ensures Assumption 4.

We also assume that the observer is designed so that, denoting $\tilde{\zeta}=z-\bar{z}$, there exists $\beta \in \mathcal{K} \mathcal{L}$ so that for any $\tilde{\zeta}_{0} \in \mathbb{R}^{n_{\bar{q}}}$ along solutions to (2), (4) this holds:

$$
|\tilde{\zeta}(t)| \leq \beta\left(\left|\tilde{\zeta}_{0}\right|, t-t_{0}\right) \quad \forall t \geq t_{0} \geq 0 .
$$

Property (29) ensures that the manifold $\mathcal{M}=\{(\eta, y, \bar{q})$ $\left.\in \mathbb{R}^{n_{\eta}+n_{y}+n_{\bar{q}}}: T_{P}(\eta, y)=T_{O}(\bar{q}, y)\right\}$ is positively invariant and globally attractive for system (2), (4) and thus observer (4) is an (immersion and invariance) observer according to Definition 1 in [9].

We now consider the scenario where the system measurements are transmitted through a shared communication channel governed by a Lyapunov UGAS protocol. We write the problem in $(\zeta, \chi, e)$ coordinates and obtain system (10)-(16). We use in-network processing algorithms that are such that $\hat{f}_{y}$ is locally Lipschitz which is the case of the zero-order-hold devices $\hat{y}$ (i.e. $\hat{f}_{y}=0$ ) or the predictive-type algorithm of [8] (i.e. $\left.\hat{f}_{y}(\bar{q}, \hat{y})=f_{y}\left(T_{P}^{L}(\bar{z}, \hat{y}), \hat{y}\right)\right)$ in view of the assumed conditions. The following corollary is a direct application of Theorem 10.

Corollary 15 Consider system (10)-(16) and suppose the following holds.

(i) Assumption 14 holds.

(ii) Protocol (15) is Lyapunov UGAS with a Lyapunov function $W(i, e)$ continuous in e, uniformly in $i$.

(iii) Function $\hat{f}_{y}$ is locally Lipschitz.

(iv) System (11) is UGS.

Then properties (22)-(24) are satisfied.

Proof. Condition (i), (iv) and (v) of Theorem 10 are satisfied respectively in view of item (i) of Corollary 15, (29) and item (iv) of Corollary 15. According to the expression of $g$ given in Section 3.2, condition (ii) of Theorem 9 is satisfied since both $\hat{f}_{y}$ and $f_{y}$ are continuous as they are locally Lipschitz from item (iii) of Corollary 15. Thus, since item (ii) of Corollary 15 holds, condition (ii) of Theorem 10 is verified. Similarly, in view of the definition of $f_{\zeta}$ given in Section 3.2, we see that it is locally Lipschitz according to Assumption 14 and item (iii) of Corollary 15. Item (iii) of Theorem 10 is therefore ensured. Since all conditions of Theorem 10 hold, the desired results are obtained.

\subsection{Circle-criterion observers}

In this subsection, we show how Theorem 13 can be used for designing a circle-criterion observer [2] for NCS in the case where the plant is only supposed to be uniformly forward complete, by using an appropriate in-network processing algorithm. Consider system (5)-(6) where $\gamma=$ $\left(\gamma_{1}, \ldots, \gamma_{r}\right)$ satisfies for all $a, b \in \mathbb{R}_{>0}$ and $i \in\{1, \ldots, r\}$ : $(a-b)\left[\gamma_{i}(a)-\gamma_{i}(b)\right] \geq 0$. An additional assumption is taken on the nonlinear functions $\rho_{1}, \rho_{2}$ and $\gamma$, similar to Assumption 6 in [17].

Assumption 16 There exist $\theta_{\rho_{1}}, \theta_{\rho_{2}}, \theta_{\gamma} \in \mathcal{K}$, with $\theta_{\gamma}$ locally Lipschitz, such that for a, $b$ of appropriate dimensions, this holds: $\left|\rho_{1}(a)-\rho_{1}(b)\right| \leq \theta_{\rho_{1}}(|a-b|), \mid \rho_{2}(a)-$ $\rho_{2}(b) \mid \leq \theta_{\rho_{2}}(|a-b|)$ and $|\gamma(a)-\gamma(b)| \leq \theta_{\gamma}(|a-b|)$. 
Assumption 16 is equivalent to saying that functions $\rho_{1}$, $\rho_{2}$ and $\gamma$ are uniformly continuous (see Proposition A.2.1 in [14]). We build the reduced-order observer (7)-(8) and we assume there exist real matrices $P$, that is symmetric positive definite, $\Lambda$, that is diagonal and positive definite, and constant $\nu>0$ such that the following LMI holds:

$$
\left[\begin{array}{cc}
\left(A_{2}+N A_{1}\right)^{\mathrm{T}} P+P\left(A_{2}+N A_{1}\right)+\nu \mathbb{I} & \star \\
\left(G_{2}+N G_{1}\right)^{\mathrm{T}} P+\Lambda H_{2} & 0
\end{array}\right] \leq 0,
$$

where $\star$ stands for $P\left(G_{2}+N G_{1}\right)+H_{2}^{\mathrm{T}} \Lambda$. We introduce $z=\eta+N y$ and $\bar{z}=\bar{q}$, i.e. $T_{P}(t, \eta, y)=\eta+N y$ and $T_{O}(t, \bar{q}, y)=\bar{q}$ as in Example 5 . The system measurements are now sent through a shared channel and we implement the observer (7)-(8) using the predictivetype in-network algorithm: $\hat{f}_{y}(\hat{y}, \bar{z})=A_{1} \bar{\eta}+G_{1} \gamma\left(H_{1} \hat{y}+\right.$ $\left.H_{2} \bar{\eta}\right)+\rho_{1}(\hat{y})$ where $\bar{\eta}=\bar{q}-N \hat{y}=\bar{z}-N \hat{y}$ in view of Example 5. We write the problem in the coordinates $(\zeta, \chi, e)$ :

$$
\begin{aligned}
\dot{\zeta}= & \left(A_{2}+N A_{1}\right) \zeta+\left(G_{2}+N G_{1}\right)\left(\gamma\left(\mu_{1}\right)-\gamma\left(\mu_{2}\right)\right) \\
& +\bar{\rho}(y)-\bar{\rho}(y+e) \quad \forall t \in\left[t_{i-1}, t_{i}\right](31) \\
\dot{\chi}= & {\left[\begin{array}{cc}
A_{2}(z-N y)+G_{2} \gamma\left(\mu_{1}\right)+\rho_{2}(y) \\
+N\left[A_{1}(z-N y)+G_{1} \gamma\left(\mu_{1}\right)+\rho_{1}(y)\right]
\end{array}\right] } \\
& \forall t \in\left[t_{i-1}, t_{i}\right](32) \\
A_{1}(z-N y)+G_{1} \gamma\left(\mu_{1}\right)+\rho_{1}(y) & \forall t \in\left[t_{i-1}, t_{i}\right](33) \\
& +\rho_{1}(y+e)-\rho_{1}(y) \\
\zeta\left(t_{i}^{+}\right)= & \zeta\left(t_{i}\right) \\
\chi\left(t_{i}^{+}\right)= & \chi\left(t_{i}\right) \\
e\left(t_{i}^{+}\right)= & h\left(i, e\left(t_{i}\right)\right) \\
\xi= & \zeta+N e,
\end{aligned}
$$

where $h$ is defined by the protocol and $\mu_{1}=H_{1} y+$ $H_{2}(z-N y)$ and $\mu_{2}=H_{2}(z-\zeta)+\left(H_{1}-H_{2} N\right)(y+e)$. The following result follows from Theorem 13.

Corollary 17 Consider system (31)-(37). Suppose the following holds.

(i) Assumption 16 holds.

(ii) Protocol (36) is Lyapunov UGAS with a Lyapunov function $W(i, e)$ continuous in e, uniformly in $i$.

(iii) Plant (5)-(6) is uniformly forward complete.

Then properties (22)-(24) are satisfied.

Proof. We show that the conditions of Theorem 13 are ensured before applying it. Condition (i) of Theorem 13 and the conditions of Lemma 11 hold with $\theta_{P}(s)=$ $\tilde{\theta}_{P}(s)=\vartheta_{P}(s)=s$ and $\bar{\theta}_{P}(s)=\overline{\tilde{\theta}}_{P}(s)=\bar{\vartheta}_{P}(s)=|N| s$ for any $s \geq 0$. Therefore, in view item (iii) of Corollary 17 and Lemma 11, condition (vi) of Theorem 13 is verified. On the other hand, item (ii) of Corollary 17 corresponds to condition (ii) of Theorem 13. According to Section 2 in [2], condition (v) of Theorem 13 is satisfied. In view of Assumption 16, it can be shown that condition (iii) of Theorem 13 holds with $\bar{g}:(\zeta, e) \mapsto\left|A_{1}\right||\zeta|+\left|A_{1} N\right||e|+$ $\left|G_{1}\right| \theta_{\gamma}\left(\left|H_{2} N-H_{1}\right||e|+\left|H_{2}\right||\zeta|\right)+\theta_{\rho_{1}}(|e|)$ which is continuous in $\zeta, e$ and so bounded on compact sets. Similarly, condition (iv) is ensured by using Assumption 16. The proof is completed by applying Theorem 13 .

\section{Conclusion}

We have shown that the global asymptotic convergence of a class of nonlinear reduced-order observers in the absence of network is maintained semiglobally and practically, with respect to the MATI, when the system measurements are transmitted through a serial communication channel governed by a Lyapunov UGAS protocol, under mild conditions. The proposed conditions have been used to synthesize immersion and invariance observers and circle criterion observers for NCS.

\section{References}

[1] D. Angeli and E.D. Sontag. Forward completeness, unboundedness observability, and their Lyapunov characterizations. Systems \& Control Letters, 38:209-217, 1999.

[2] M. Arcak and P. Kokotović. Nonlinear observers: a circle criterion design and robustness analysis. Automatica, 37:1923-1930, 2001.

[3] A. Astolfi, D. Karagiannis, and R. Ortega. Nonlinear and Adaptive Control with Applications. Springer, New York, U.S.A., 2008.

[4] E.D. Sontag D. Angeli and Y. Wang. A characterization of integral input-to-state stability. IEEE Transactions on Automatic Control, 45(6):1082-1097, 2000.

[5] J.P. Gauthier, H. Hammouri, and S. Othman. A simple observer for nonlinear systems - applications to bioreactors. IEEE Transactions on Automatic Control, 37:875-880, 1992.

[6] Z.-P. Jiang, A.R. Teel, and L. Praly. Small-gain theorem for ISS systems and applications. Mathematics of Control, Signals, and Systems, 7:95-120, 1994.

[7] P. Jouan. Immersion of nonlinear systems into linear systems modulo output injection. SIAM Journal on Control and Optimization, 41(6):1756-1778, 2003.

[8] I. Karafyllis and C. Kravaris. From continuous-time design to sampled-data design of observers. IEEE Transactions on Automatic Control, 54(9):2169-2174, 2009.

[9] D. Karagiannis, D. Carnevale, and A. Astolfi. Invariant manifold based reduced-order observer design for nonlinear systems. IEEE Transactions on Automatic Control, 53(11):2602-2614, 2008.

[10] H.K. Khalil and F. Esfandiari. Semiglobal stabilization of a class of nonlinear systems using output feedback. IEEE Transactions on Automatic Control, 38(9):1412-1415, 1993.

[11] A. J. Krener and A. Isidori. Linearization by output injection and nonlinear observers. Systems \& Control Letters, 3:47-52, 1983.

[12] D. Nešić and A.R. Teel. Input-output stability properties of networked control systems. IEEE Transactions on Automatic Control, 49:1650-1667, 2004. 
[13] D. Nešić and A.R. Teel. Input-to-state stability of networked control systems. Automatica, 40:2121-2128, 2004.

[14] R. Postoyan. Commande et construction d'observateurs pour les systèmes non linéaires à données échantillonnées et en réseau. PhD thesis, Univ Paris-Sud (in French), 2009.

[15] R. Postoyan, T. Ahmed-Ali, and F. Lamnabhi-Lagarrigue. Observers for classes of nonlinear networked systems. In SSD'09 (IEEE International Conference on Systems, Signals and Devices), Djerba, Tunisia, 2009.

[16] R. Postoyan and D. Nešić. A framework for the observer design for networked control systems. In American Control Conference, Baltimore, U.S.A., pages 3678-3683, 2010.

[17] R. Postoyan and D. Nešić. On emulation-based observer design for networked control systems. In CDC (IEEE Conference on Decision and Control) Atlanta, U.S.A., 2010.

[18] G.C. Walsh, O. Beldiman, and L.G. Bushnell. Asymptotic behavior of nonlinear networked control systems. IEEE Transactions on Automatic Control, 46:1093-1097, 2001.

\section{A Appendix}

\section{A.1 Proofs of the main results}

Proof of Theorem 9. The proof follows the same lines than the proof of Theorem 1 in [13] by identifying $x=$ $(\zeta, \chi)$. The only difference is that Theorem 1 (and Proposition 3) in [13] makes use of the uniform continuity ${ }^{2}$ of $W$ in $e$ whereas here $W$ is only assumed to be continuous in $e$, uniformly in $i$. According to Lemma 19, by identifying $f(x, y)=W(i, e)$ and $g(y)=\bar{\alpha}(|e|)$ (where $\bar{\alpha}$ comes from (17)), since $W$ is continuous in $e$, uniformly in $i$ and $\bar{\alpha}$ is continuous, there exist $\bar{\varepsilon}, \tilde{\varepsilon} \in \mathcal{K}_{\infty}$ such that, for any $c \in \mathbb{R}_{>0}, i \in \mathbb{R}_{>0}, e_{1}, e_{2} \in \mathbb{R}^{n_{e}}$ with $\max \left\{\left|e_{1}\right|,\left|e_{2}\right|\right\} \leq c$, this holds: $W\left(i, e_{1}\right)-W\left(i, e_{2}\right) \leq$ $\bar{\varepsilon}(c) \tilde{\varepsilon}\left(\left|e_{1}-e_{2}\right|\right)$. On the other hand, from item (ii) of Theorem 9, there exists a continuous positive function $\varphi: \mathbb{R}_{>0} \rightarrow \mathbb{R}_{>0}$ (not necessarily zero at zero), strictly increasing and such that $\lim _{s \rightarrow \infty} \varphi(s)=\infty$ that satisfies: $|g(t, \zeta, \chi, e, w)| \leq \varphi(\max \{|\zeta|,|\chi|,|e|,|w|\})$ for any $(\zeta, \chi, e, w) \in \mathbb{R}^{n_{\zeta}+n_{\chi}+n_{e}+n_{w}}$ and $t \in \mathbb{R}_{\geq 0}$. We define $\tau_{1}^{*}:(\varepsilon, c) \mapsto \frac{1}{1+\varphi\left(\max \left\{c+1, \alpha_{1}^{-1}(c+1)\right\}\right)} \tilde{\varepsilon}^{-1}\left(\frac{\varepsilon}{\bar{\varepsilon}(c+1)}\right)$ that is of class $\mathcal{K} \mathcal{L}$. Afterwards, by following the same lines than the proof of Proposition 3 and Theorem 1 in [13] and using the last inequality of Lemma 18 to convert maximum inequalities into a sum, the desired result is obtained. $\square$

Proof of Theorem 10. Consider system (10)(16). From Lemma 1 and Proposition 4 in [13], by identifying $x=\zeta, w=(\chi, w), e=e, \beta=\beta_{2}$,

\footnotetext{
2 Indeed, there is an error in the statement of item (1) of Theorem 1 (equivalently item (1) of Proposition 3) in [13]: $W$ must be uniformly continuous in $e$ (and not only continuous in $e)$ in order to have $W\left(i, e_{1}\right)-W\left(i, e_{2}\right) \leq \tilde{\varepsilon}\left(\left|e_{1}-e_{2}\right|\right)$ with $\tilde{\varepsilon} \in \mathcal{K}_{\infty}$ for $i \in \mathbb{Z}_{>0}, e_{1}, e_{2} \in \mathbb{R}^{n_{e}}$ in the proof of Proposition 3. Nonetheless, a similar inequality can be obtained by restricting $e_{1}$ and $e_{2}$ to belong to a given compact set as shown here.
}

$\gamma\left(\|w\|_{\infty}\right)=\gamma_{2}\left(\|w\|_{\infty}\right)$, all required conditions hold (the fact that (21) is stated as a sum and not a maximum as in (13) in [13] has no incidence on the proofs of the cited results), so there exists $\bar{\tau} \in \mathcal{K} \mathcal{L}$ such that for any $\varepsilon, \Delta \in \mathbb{R}_{>0}, \tau \in[v, \bar{\tau}(\varepsilon, \Delta))$ and $\max \left\{\left|\zeta_{0}\right|,\|e\|_{\infty},\|w\|_{\infty},\|\chi\|_{\infty}\right\}<\Delta$, the following inequality holds, for any $t \geq t_{0} \geq 0$ :

$$
|\zeta(t)| \leq \beta_{2}\left(\left|\zeta_{0}\right|, t-t_{0}\right)+\gamma_{2}\left(\|w\|_{\infty}\right)+\varepsilon .
$$

Consequently, by using similar arguments than in the proof of Theorem 2.1 in [6] (as for Theorem 2 in [13]), we obtain that there exists $\tau^{*} \in \mathcal{K} \mathcal{L}$ such that, for any $\varepsilon, \Delta \in \mathbb{R}_{>0}, \tau \in\left[v, \tau^{*}(\varepsilon, \Delta)\right)$, $\max \left\{\left|\zeta_{0}\right|,\left|e_{0}\right|,\|w\|_{\infty},\|\chi\|_{\infty}\right\}<\Delta$, this holds, for any $t \geq t_{0} \geq 0$ :

$$
\begin{aligned}
|e(t)| & \leq \beta_{1}\left(\left|e_{0}\right|, \frac{t-t_{0}}{\tau}\right)+\varepsilon \\
|\zeta(t)| & \leq \beta_{2}\left(\left|\zeta_{0}\right|, t-t_{0}\right)+\gamma_{2}\left(\|w\|_{\infty}\right)+\varepsilon .
\end{aligned}
$$

We now need to relax the requirement that $\|\chi\|_{\infty}<\Delta$ in (A.2)-(A.3) to $\left|\chi_{0}\right| \leq \Delta$ to show that (22) and (23) are satisfied. We use for that condition (v) of Theorem 10. Indeed, in view of condition (v) of Theorem 10 and Definition 1 , there exist $K, \gamma \in \mathcal{K}$ such that for any $\chi_{0} \in \mathbb{R}^{n_{\chi}}$ and $w \in \mathcal{L}_{\infty}$, the following holds for any $t \geq t_{0} \geq 0:|\chi(t)| \leq K\left(\left|\chi_{0}\right|\right)+\gamma\left(\|w\|_{\infty}\right)$, thus, defining $\alpha: s \mapsto \max \{s,(K+\gamma)(s)\} \in \mathcal{K}_{\infty}$, for any $\Delta \in \mathbb{R}_{>0}$, $\left|\chi_{0}\right|<\Delta$ and $\|w\|_{\infty}<\Delta$ we have that:

$$
\|\chi\|_{\infty} \leq(K+\gamma)(\Delta) \leq \alpha(\Delta) .
$$

From this condition, we could show that (22) and (23) are verified by appropriately defining the MATI depending on $(\varepsilon, \Delta)$. Nevertheless, because we want to consider the same MATI for (22)-(24), we anticipate from the sequel and do the following. Let $\varepsilon, \Delta \in \mathbb{R}_{>0}$ and define $\theta: s \mapsto \min \left\{s, \tilde{\theta}^{-1}(s)\right\} \in \mathcal{K}_{\infty}$ with $\tilde{\theta}: s \mapsto \theta_{P}(4 s)+$ $\bar{\theta}_{P}(2 s) \in \mathcal{K}_{\infty}$ (where $\theta_{P}$ and $\bar{\theta}_{P}$ come from Assumption 4). We denote $\tilde{\tau}:(r, s) \mapsto \tau^{*}(\theta(r), \alpha(s)) \in \mathcal{K} \mathcal{L}$. Let $\tau \in[v, \tilde{\tau}(\varepsilon, \Delta)),\left(\zeta_{0}, \chi_{0}, e_{0}\right) \in \mathbb{R}^{n_{\zeta}+n_{\chi}+n_{e}}$ and $w \in \mathcal{L}_{\infty}$ be such that $\max \left\{\left|\zeta_{0}\right|,\left|e_{0}\right|,\left|\chi_{0}\right|,\|w\|_{\infty}\right\}<\Delta \leq \alpha(\Delta)$. In view of (A.4), we have that $\max \left\{\left|\zeta_{0}\right|,\left|e_{0}\right|,\left|\chi_{0}\right|,\|w\|_{\infty}\right\} \leq$ $\alpha(\Delta)$ and the following holds for any $t \geq t_{0} \geq 0$ :

$$
\begin{aligned}
|e(t)| & \leq \beta_{1}\left(\left|e_{0}\right|, \frac{t-t_{0}}{\tau}\right)+\theta(\varepsilon) \\
& \leq \beta_{1}\left(\left|e_{0}\right|, \frac{t-t_{0}}{\tau}\right)+\varepsilon \\
|\zeta(t)| & \leq \beta_{2}\left(\left|\zeta_{0}\right|, t-t_{0}\right)+\gamma_{2}\left(\|w\|_{\infty}\right)+\varepsilon,
\end{aligned}
$$

so (22) and (23) are ensured. We now show that (24) is guaranteed. Consider variable $\xi$, we have $\xi=T_{P}^{L}(t, z, y)-T_{P}^{L}(t, z-\zeta, y+e)$. According to Assumption 4, this holds: $|\xi| \leq \theta_{P}(|\zeta|)+\bar{\theta}_{P}(|e|)$, consequently, from (A.5), (A.6) and using Lemma 18, the definition of 
$\tilde{\theta}$ and the facts that $\tau<\tilde{\tau}(\varepsilon, \Delta)=\tau^{*}(\theta(\varepsilon), \alpha(\Delta))$, the following is satisfied for any $t \geq t_{0} \geq 0$ :

$$
\begin{aligned}
|\xi(t)| \leq & \theta_{P}(|\zeta(t)|)+\bar{\theta}_{P}(|e(t)|) \\
\leq & \theta_{P}\left(\beta_{2}\left(\left|\zeta_{0}\right|, t-t_{0}\right)+\gamma_{2}\left(\|w\|_{\infty}\right)+\theta(\varepsilon)\right) \\
& +\bar{\theta}_{P}\left(\beta_{1}\left(\left|e_{0}\right|, \frac{t-t_{0}}{\tau}\right)+\theta(\varepsilon)\right) \\
\leq & \theta_{P}\left(2 \beta_{2}\left(\left|\zeta_{0}\right|, t-t_{0}\right)\right)+\theta_{P}\left(4 \gamma_{2}\left(\|w\|_{\infty}\right)\right)+ \\
& +\bar{\theta}_{P}\left(2 \beta_{1}\left(\left|e_{0}\right|, \frac{t-t_{0}}{\tau}\right)\right)+\tilde{\theta}(\theta(\varepsilon)) . \quad \text { (A.7) }
\end{aligned}
$$

Since $\theta(\varepsilon) \leq \tilde{\theta}^{-1}(\varepsilon)$, we get from (A.7) that: $|\xi(t)| \leq$ $\beta\left(\left|\left(\zeta_{0}, e_{0}\right)\right|, t-t_{0}\right)+\gamma\left(\|w\|_{\infty}\right)+\varepsilon$, with $\beta:(s, t) \mapsto$ $\theta_{P}\left(2 \beta_{2}(s, t)\right)+\bar{\theta}_{P}\left(2 \beta_{1}\left(s, \frac{t}{\tau}\right)\right) \in \mathcal{K} \mathcal{L}$ and $\gamma: s \mapsto$ $\theta_{P}\left(4 \gamma_{2}(s)\right) \in \mathcal{K}$ : property $(24)$ is guaranteed.

Proof of Theorem 13 (Sketch). The proof follows the same lines as the proof of Theorem 10. First, we show that (19) is satisfied for any $\chi \in \mathcal{L}_{l o c, \infty}$. In the proof of Proposition 3 in [13], instead of using the fact that $g$ is bounded on any compact sets, uniformly in $t$, we invoke condition (iii) of Theorem 13 and define the function $\varphi$ in the proof of Theorem 9 by bounding $\bar{g}$ (and not $g$ ). Afterwards, by invoking the same arguments as the proof of Theorem 10 but with no boundedness condition on $\|\chi\|$ and the fact that system (22) is uniformly forward complete (according to item (vi) of Theorem 13), we obtain the desired results.

\section{A.2 Technical lemmas}

Lemma 18 For any $\gamma \in \mathcal{K}$ and $a, b \in \mathbb{R}_{\geq 0}$, this holds: $\gamma(a+b) \leq \gamma(2 a)+\gamma(2 b), a+b \leq \max \{2 a, 2 b\}$ and $\max \{a, b\} \leq a+b$.

Lemma 19 Let $f:(x, y) \mapsto f(x, y)$ from $\mathbb{R}^{n+m}$ to $\mathbb{R}^{p}$ be continuous in $y$, uniformly in $x$, and such that $|f(x, y)| \leq$ $g(y)$ for any $(x, y) \in \mathbb{R}^{n+m}$ with $g \in C\left(\mathbb{R}^{m}, \mathbb{R}_{\geq 0}\right)$. There exist $\gamma_{1}, \gamma_{2} \in \mathcal{K}_{\infty}$ such that for any $c \in \mathbb{R}_{\geq 0},\left(x, y_{1}, y_{2}\right) \in$ $\mathbb{R}^{n+2 m}$ with $\max \left\{\left|y_{1}\right|,\left|y_{2}\right|\right\} \leq c$, this holds: $\mid f\left(x, y_{1}\right)-$ $f\left(x, y_{2}\right) \mid \leq \gamma_{1}(c) \gamma_{2}\left(\left|y_{1}-y_{2}\right|\right)$.

Proof. For any $c, s \geq 0$, define $\mathcal{D}(c, s)=\left\{\left(y_{1}, y_{2}\right) \in\right.$ $\left.\mathbb{R}^{2 m}:\left|y_{1}-y_{2}\right| \leq s, \max \left\{\left|y_{1}\right|,\left|y_{2}\right|\right\} \leq c\right\}$. Let $\psi(c, s)=\quad \sup _{\log }\left\{\left|f\left(x, y_{1}\right)-f\left(x, y_{2}\right)\right|\right\}$ for $c, s \geq 0$. Function $\psi$ is well defined, since for any $c, s \geq 0: \psi(c, s) \leq \sup _{\left(y_{1}, y_{2}\right) \in \mathcal{D}(c, s)}\left\{g\left(y_{1}\right)+g\left(y_{2}\right)\right\} \in \mathbb{R}_{\geq 0}$. Moreover, since $f$ is continuous in $y$, uniformly in $x, \psi$ is continuous in $c, s$. In addition, $\psi$ is non-decreasing in $c$ and $s$. For any $c \in \mathbb{R}_{\geq 0},\left(x, y_{1}, y_{2}\right) \in \mathbb{R}^{n+2 m}$ with $\max \left\{\left|y_{1}\right|,\left|y_{2}\right|\right\} \leq c$, the following holds:

$$
\left|f\left(x, y_{1}\right)-f\left(x, y_{2}\right)\right| \leq \psi\left(c,\left|y_{1}-y_{2}\right|\right) .
$$

Let introduce $\tilde{\psi}(c, s)=\psi(c, s)+c s$ for $c, s \geq 0$, in view of the properties of $\psi, \tilde{\psi}(c, \cdot) \in \mathcal{K}_{\infty}$ and $\tilde{\psi}(\cdot, s) \in \mathcal{K}_{\infty}$ for $c, s \in \mathbb{R}_{>0}$. Additionally, according to (A.8) and the definition of $\tilde{\psi}$, this holds for any $c \in \mathbb{R}_{>0},\left(x, y_{1}, y_{2}\right) \in$ $\mathbb{R}^{n+2 m}$ with $\max \left\{\left|y_{1}\right|,\left|y_{2}\right|\right\} \leq c$ :

$$
\left|f\left(x, y_{1}\right)-f\left(x, y_{2}\right)\right| \leq \tilde{\psi}\left(c,\left|y_{1}-y_{2}\right|\right) .
$$

Invoking Corollary IV.5 in [4], we know that there exist $\gamma_{1}, \gamma_{2} \in \mathcal{K}_{\infty}$ such that, for any $c, s \geq 0$, this holds: $\tilde{\psi}(c, s) \leq \gamma_{1}(c) \gamma_{2}(s)$. As a consequence, in view of (A.9), for any $c \in \mathbb{R}_{\geq 0},\left(x, y_{1}, y_{2}\right) \in \mathbb{R}^{n+2 m}$ with $\max \left\{\left|y_{1}\right|,\left|y_{2}\right|\right\} \leq c$, this holds: $\left|f\left(x, y_{1}\right)-f\left(x, y_{2}\right)\right| \leq$ $\gamma_{1}(c) \gamma_{2}\left(\left|y_{1}-y_{2}\right|\right)$. This completes the proof. 